\title{
Using seismic waves to image Earth's internal structure
}

\author{
Barbara Romanowicz
}

\section{Seismic waves generated in Earth's interior provide images that help us to better understand the pattern of mantle convection that drives plate motions.}

Forty years after the discovery of seafloor spreading and the acceptance of the theory of plate tectonics, important gaps remain in our understanding of the pattern of convection that drives the motions of the plates, leading to earthquakes, tsunamis and volcanic eruptions. There are still many heated debates. Does oceanic lithosphere pushed down into the interior at converging plate boundaries reach the bottom of Earth's mantle? Do deep-rooted, thin hot plumes rise through the mantle under mid-plate 'hot spot' volcanoes? What is the relative importance of compositional versus thermal heterogeneity in mantle convection? And what role does Earth's solid inner core have in the 'geodynamo', which keeps Earth's magnetic field alive, and in the thermal evolution of our planet (see page 261)? To address these controversies, seismology has been brought to bear to image Earth's deep interior. From the construction of accurate models of Earth's one-dimensional radial structure (Fig. 1) to the current models of its three-dimensional structure (Fig. 2), progress in seismic imaging has gone hand in hand with improvements in the design of seismic sensors, the capacity to record digitally increasingly massive quantities of data, theoretical progress in handling seismic-wave propagation through complex three-dimensional media and the development of powerful computers for simulating seismic waves and for the inversion of large matrices.

From seismic tomography, first introduced in the late 1970 s (refs 1,2), we now have a good understanding of the first-order characteristics of the long-wavelength $(\sim 1,000-2,000 \mathrm{~km})$ three-dimensional elastic structure of Earth's mantle ${ }^{3}$. At shorter wavelengths $(\sim 200 \mathrm{~km})$, fast-velocity 'slabs' representing oceanic lithosphere plunging back into the mantle are, today, the best-resolved 'objects', because of the favourable geometry; many earthquake sources illuminate such slabs from both below and above, at least down to $\sim 600 \mathrm{~km}$ depth (Fig. 3a). It is tempting to interpret the large-scale features imaged throughout the mantle in terms of lateral variations in temperature, which can be as much as several hundred degrees Celsius. For example, the fast ring of high velocities at the bottom of the mantle (shown in blue in Fig. 2) might well represent the 'graveyard' of cold subducted lithosphere, and the slow regions, commonly referred to as 'superplumes', the hot rising return flow (shown in red in Fig. 2). It is increasingly clear, however, that compositional variations also have an important role in mantle convection.

With the deployment, starting in the early 1980s, of high-quality digital broadband seismic stations around the world (Fig. 3b), finerscale imaging became possible. Particularly striking is the accumulating evidence for complexity in the lower $300-400 \mathrm{~km}$ of the mantle, the so-called $\mathrm{D}^{\prime \prime}$ region, an important chemical and thermal boundary layer. Many intriguing seismic observations have been made in this region $^{4,5}$, including the remarkable observation that the lateral transition from fast shear velocity regions in $\mathrm{D}^{\prime \prime}$ into the superplumes occurs abruptly, over a much smaller range than would be possible if lateral variations in temperature were the only cause ${ }^{6}$. Perhaps less surprisingly, closer to Earth's surface such strong lateral contrasts are also found at lithospheric depths, especially at the edges of tectonic provinces of different origin and age.

Characterizing the sharpness or fuzziness of the boundaries of the heterogeneous structures deep inside the planet, and detecting and mapping small-scale heterogeneity, are the next steps. This will mean extracting more information from seismograms than has traditionally been done. Indeed, neither remnants of compositionally distinct lithosphere in the lower mantle nor narrow plume conduits (if they exist) can be accurately mapped by standard tomographic approaches that make use only of information carried by the most direct waves - those that travel along the shortest paths - according to the simple

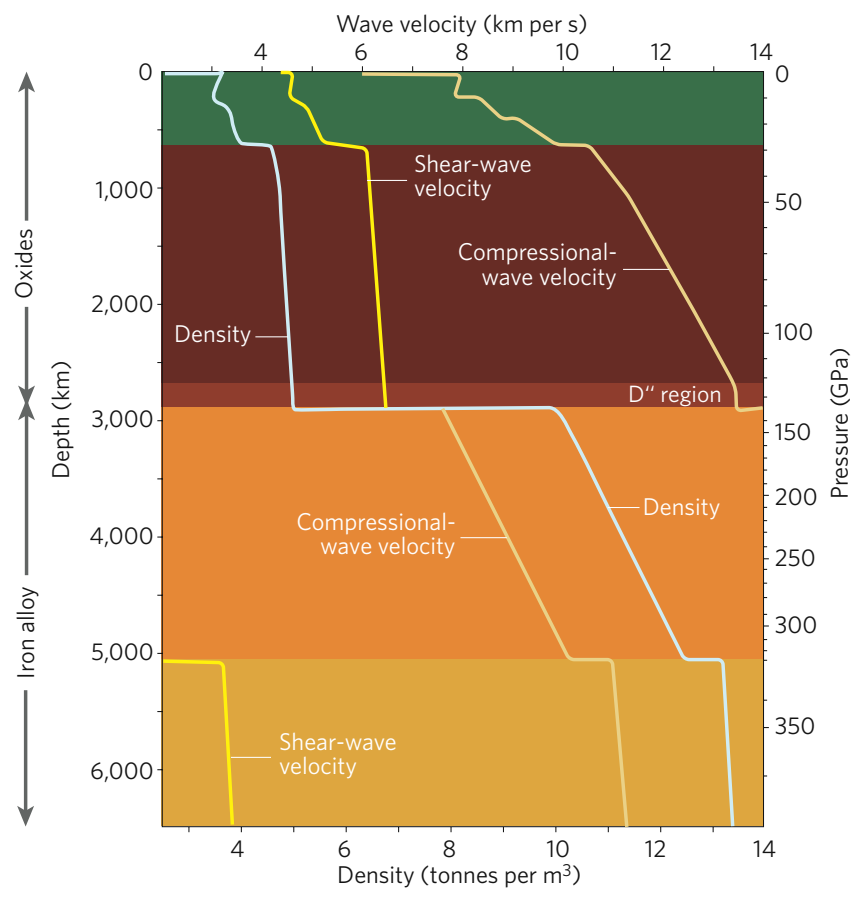

Figure 1 | Radial structure of Earth. The first-order structural units of Earth - its suite of concentric shells and their approximate composition - were established over the first half of the twentieth century from measurements of the travel times of seismic waves refracted and reflected inside Earth, whereas proof of the solidity of the inner core had to await the capability to record and digitize long time series and measure the frequencies of free oscillations. The ' $660 \mathrm{~km}$ ' discontinuity is a phase change, and possibly a compositional change, in the silicate mantle. This illustration is of the preliminary reference Earth model ${ }^{14}$. 
$140 \mathrm{~km}$
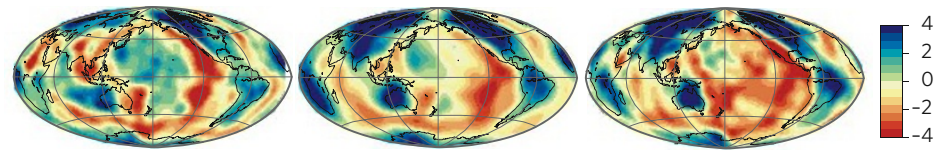

$925 \mathrm{~km}$
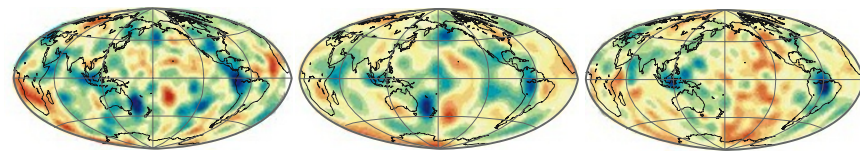

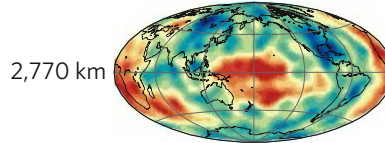

SAW24B16

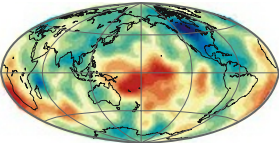

S362D1

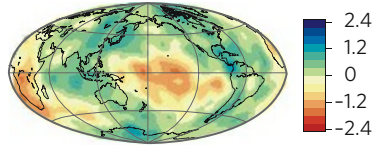

S2ORTS

Figure 2 | Large-scale three-dimensional Earth structure as inferred from seismic tomography. Each column of images represents a different model of mantle shear-velocity structure (using various data sources) shown at three representative depths $(140 \mathrm{~km}, 925 \mathrm{~km}$ and $2,770 \mathrm{~km}$ ). Left, SAW24B16, developed at the University of California at Berkeley ${ }^{15}$; centre, S362D1, developed at Harvard University ${ }^{16}$; and right, S20RTS, developed as a collaboration between the University of Oxford and California Institute of Technology ${ }^{17}$. In the top $\sim 250 \mathrm{~km}$ of the mantle, the structure follows the surface tectonics: slow ridges and back-arcs (red), fast roots under stable continents (blue) and a progressively faster velocity away from mid-ocean ridges, consistent with expectations from a simple cooling plate model. Below the thickest lithospheric roots $(250 \mathrm{~km})$, the pattern changes, and in the transition zone a clear signature of fast anomalies associated with subducted slabs emerges. Recent models show a variety of behaviours for these slabs: some seem to be stagnating in the upper mantle; for others, the fast-velocity anomaly seems to continue at oblique or steep angles into the lower mantle. Two regions, in northwestern America and Southeast Asia, show fast-velocity anomalies that may be related to past subduction down to considerable depths $(\sim 1,200-1,400 \mathrm{~km})$. In the mid-mantle, the spectrum of heterogeneity becomes white, which indicates that it is dominated by smaller-scale features. In the bottom $1,000 \mathrm{~km}$ of the mantle, as we approach the core-mantle boundary, a new pattern of long-wavelength heterogeneity progressively emerges, with two very large antipodal low-velocity regions centred in the Pacific Ocean and under Africa and surrounded by faster than average material. The units of the key are relative shear-velocity changes (as percentages) with respect to the global mean at the given depth.

rules of ray theory. It will be necessary to take account of the energy bouncing off weak scatterers that can have a wide range of sizes. In practice, this means working in a wide frequency band, at short spatial wavelengths, using both the amplitude and the travel times of all possible seismic phases - that is, the entire seismogram — and applying signal-enhancing techniques.

A significant challenge is the limited distribution of seismic-wave sources and receivers. Ideally, one would want to sample the volume of Earth uniformly. But unlike other disciplines that use imaging, such as medical tomography or petroleum exploration, earthquake seismologists cannot optimize their experimental geometry (Fig. 3). To overcome these limitations, several promising approaches are being pursued.

New and exciting horizons have recently opened up with increasing capabilities in both computation and data collection. There are now powerful numerical schemes to compute synthetic seismograms in structures of arbitrary complexity, such as the spectral element method ${ }^{7}$, which are well adapted to the spherical global geometry of Earth. They can be used in a variety of ways, for forward modelling of observed seismic waveforms, as well as for inversion of the seismogram to retrieve the three-dimensional structure. They are still heavy on computation but hold much promise for the construction of the next generation of global tomographic models. Anisotropy and dissipation, which also influence seismic-wave propagation, can now be better characterized and provide additional information on flow directions, temperature variations and the presence of partial melting. At the higher end of the seismic spectrum, the deployments of dense permanent regional arrays, such as Hi-net in Japan, or temporary ones such as those of PASSCAL (http://www.iris.edu/about/PASSCAL), are stimulating the

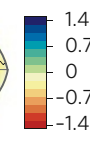

development of techniques that are beginning to erode the difference between global seismology and exploration geophysics.

Through the utilization of energy scattered both backward and forward, impressively detailed images of slabs are starting to be constructed. For the first time, it is possible to use the results of seismic imaging to trace the fate of water as it is entrained down into the mantle with the subducting slab ${ }^{8}$. The global seismic network, complemented by PASSCAL-type deployments ${ }^{9,10}$, and local dense arrays provide sufficient spatial sampling in some continental areas to investigate fine-scale layering in the deep mantle using newly developed sophisticated back-projection techniques. Much is expected from the data set now being assembled through the USArray programme of Earthscope (http://www.iris.edu/USArray). Seismologists can start to put precise constraints on velocity contrasts and the sizes and depths of heterogeneous bodies. These can be combined with experimental and theoretical data about mineral physics to determine lateral variations in composition and temperature. For example, in the case of the recently discovered post-perovskite transition, which is thought to occur in the temperature/pressure range of the $\mathrm{D}^{\prime \prime}$ region (see page 269), mineral physicists and geodynamicists are working hand in hand with seismologists to search for its presence in the deep mantle and evaluate its consequences for mantle dynamics ${ }^{4,5}$.

The approaches mentioned above assume that appropriately distributed earthquake sources are available. Where this is not possible, a rapidly developing technique to eliminate the constraints associated with natural earthquakes is building on the data set of continuous broadband waveforms accumulated by many stations in the world. Background seismic noise continuously excited by the oceans and the atmosphere can be used to construct tomographic images through noise crosscorrelation. The promise of this approach has been demonstrated in the investigation of the crust ${ }^{11}$, for which the presence of strong energy in the microseismic frequency band ( 1-15s) can be exploited. A possible extension of the technique to longer-period seismic waves presents interesting prospects for imaging the upper mantle at high resolution down to at least the base of the lithosphere.

This still leaves the oceans, where recording is limited to sparsely distributed islands. Yet there are key geodynamic problems to be addressed: for instance, the deep structure and anisotropy of ocean basins are not well understood. Most volcanic hot spots are in the oceans. The recent controversy about the 'banana-doughnut kernel' technique ${ }^{12}$ indicates the level of frustration: improvements in wave-propagation theory and inclusion of scattering effects cannot make up for the fact that stations on hot-spot islands are isolated, so that it is not possible to accurately constrain the depth and lateral extent of underlying slow anomalies. Many areas in the deep mantle and the core are currently not accessible because of a lack of stations in the oceans. Although efforts to instrument the ocean floor have been ongoing for more than 20 years, long-term ocean-floor broadband stations are still few. Local temporary deployments, such as those beneath mid-ocean ridges, have led to spectacular results ${ }^{13}$, and other ongoing projects, such as the Plume project in Hawaii, will help to address specific targets. A cabled observatory is planned in the northwest Pacific, combining Canadian and US efforts (http://www.orionprogram.org/OOI/default.html). But an internationally coordinated programme is needed to systematically deploy large-aperture $(1,000 \mathrm{~km} \times 1,000 \mathrm{~km})$ broadband ocean-floor arrays that would be left in place for at least one or two years, to record a sufficient number and variety of earthquakes and progressively fill the gap in illuminating deep structure under the oceans. 

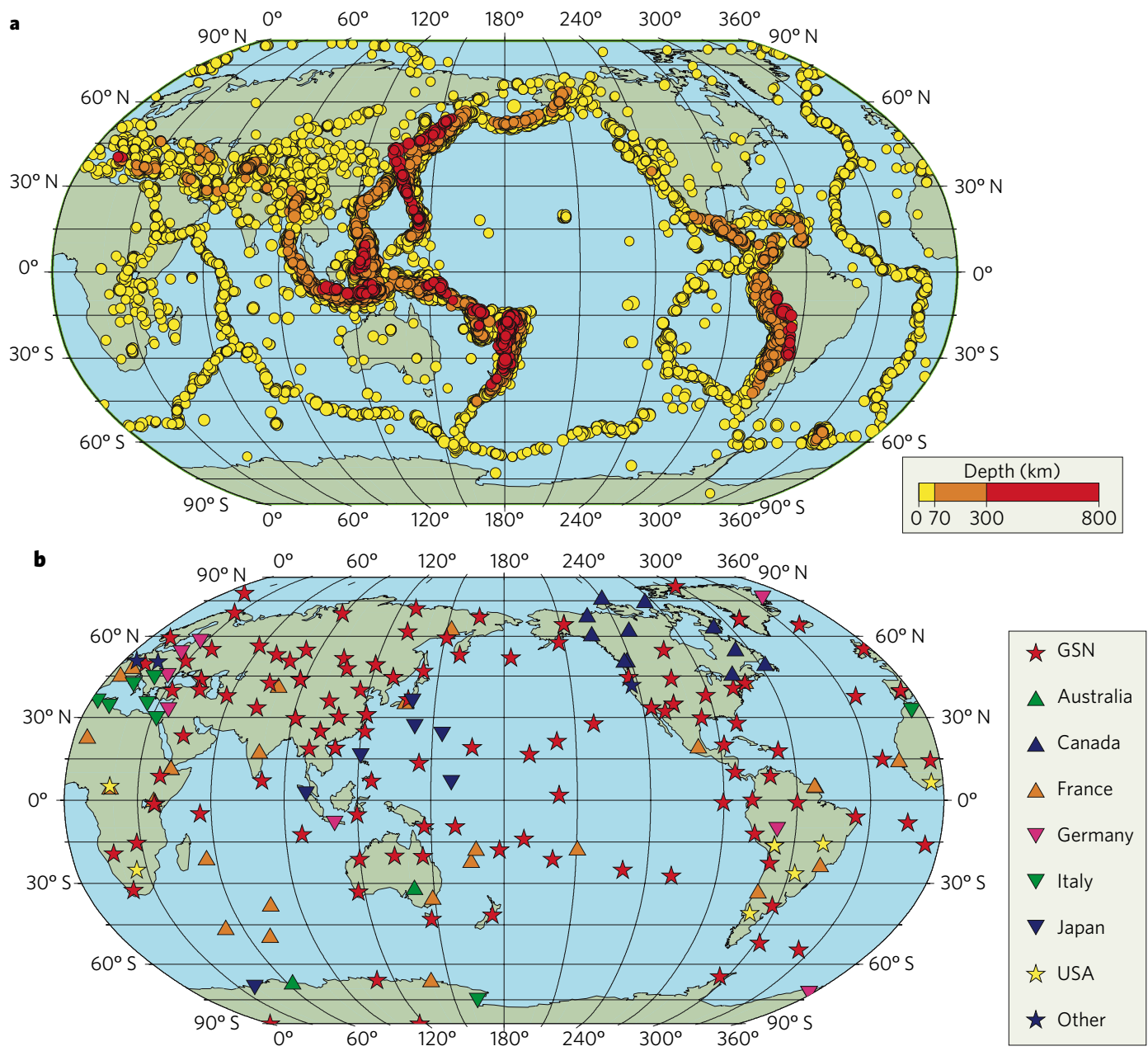

Figure 3 Global seismicity and networks. a, Worldwide distribution of earthquakes of magnitude $\left(M_{\mathrm{w}}\right)$ greater than 5.0 from 1 January 1991 to 31 December 1996. Earthquakes occur mainly along plate boundaries, delineating, in particular, the global mid-ocean ridge system. Earthquakes are generally shallow (yellow). In subduction zones around the Pacific Ocean and in the collision zones in southern Eurasia, intermediate-depth (orange) and deep (red) earthquakes indicate the presence of cold lithospheric slabs

Finally, as the images provided by seismologists become sharper, there is an increasing opportunity to work closely with other geoscientists geochemists, geodynamicists and mineral physicists - to make the best of complementary constraints for the challenging 'inverse problem' that the interior of our planet represents - that is, to use observations at or near the surface of Earth to constrain ideas about its deep structure and dynamics. Better communication and cross-education among these disciplines is key to progress. This is why interdisciplinary programmes such as the Cooperative Institute for Deep Earth Research (http://www. deep-earth.org) are needed.

Barbara Romanowicz is at the Berkeley Seismological Laboratory and the Department of Earth and Planetary Science, University of California at Berkeley, 215 McCone Hall, Berkeley, California 94720, USA.

1. Dziewonski, A. M., Hager, B. H. \& O'Connell, R. J. Large scale heterogeneities in the lower mantle. J. Geophys. Res. 82, 239-255 (1977).

2. Aki, K., Christofferson, A.\& Husebye, E. Determination of the three-dimensional structure of the lithosphere. J. Geophys. Res. 82, 277-296 (1977)

3. Romanowicz, B. Global mantle tomography: progress status in the last 10 years. Annu. Rev. Geophys. Space Phys. 31, 303-328 (2003).

4. Lay, T. et al. The core mantle boundary layer and deep mantle dynamics. Nature 392, 461-468 (1998).

5. Hirose, K. Postperovskite phase transition and its geophysical implications. Rev. Geophys. 44, RG3001,18p (2006) plunging into Earth's mantle. b, The current global broadband digital seismic network (shown as at October 2007) has been constructed through an international effort coordinated by the Federation of Digital Seismic Networks (FDSN), complemented by denser permanent regional arrays (not shown) and temporary regional deployments. GSN, Global Seismic Network (the US component of the international network). (Panel b courtesy of R. Butler, IRIS, Washington DC.)

6. Wen, L. Seismic evidence for a rapidly varying compositional anomaly at the base of the Earth's mantle beneath the Indian Ocean. Earth Planet. Sci. Lett. 194, 83-95 (2001).

7. Komatitsch, D., Ritsema, J. \& Tromp, J. The spectral element method, Beowulf computing and global seismology. Science 298, 1737-1742 (2002).

8. Kawakatsu, H. \& Watada, S. Seismic evidence for deep-water transportation in the mantle Science 316, 1468-1471 (2007).

9. Bostock, M. G. et al. An inverted continental Moho and serpentinization of the forearc mantle. Nature 417, 536-538 (2007).

10. Van der Hilst, R. et al. Seismostratigraphy and thermal structure of Earth's core-mantle boundary region. Science 315, 1813-1817 (2007).

11. Shapiro, N. et al. High resolution surface-wave tomography from ambient seismic noise. Science 307, 1615-1618 (2005).

12. Kerr, R. A. Rising plumes in Earth's mantle: phantom or real? Science 313,1726 (2006).

13. Forsyth, D. W., Webb, S. C., Dorman, L. M. \& Shen, Y. Phase velocities of Rayleigh waves in the MELT experiment on the East Pacific Rise. Science 280, 1235-1238 (1998).

14. Dziewonski, A. M. \& Anderson, D. L. Preliminary reference Earth model. Phys. Earth Planet. Inter. 25, 297-356 (1981)

15. Mégnin, C. \& Romanowicz, B. The 3D shear velocity structure of the mantle from the inversion of body surface and higher mode waveforms. Geophys. J. Int. 143, 709-729 (2000).

16. Gu, Y. J. A., Dziewonski, M., Su, W.-J. \& Ekström, G. Models of the mantle shear velocity and discontinuities in the pattern of lateral heterogeneities. J. Geophys. Res. 106, 11169-11199 (2001).

17. Ritsema, J., van Heijst, H. J. \& Woodhouse, J. H. Complex shear wave velocity structure imaged beneath Africa and Iceland. Science 286, 1925-1928 (1999).

Author Information Reprints and permissions information is available at npg.nature.com/reprints. Correspondence should be addressed to the author (barbara.romanowicz@gmail.com). 